\title{
DETERMINANTES DA SOBRECARGA E DESCONFORTO EMOCIONAL EM CUIDADORES DE IDOSOS
}

\author{
Luana Araújo dos Reis*, Kleyton Trindade dos Santos**, Nadirlene Pereira Gomes***, \\ Luciana Araújo dos Reis****
}

Autora para correspondência: Luciana Araújo dos Reis - lucianauesb@yahoo.com.br

* Mestre em Enfermagem pela Universidade Federal da Bahia e docente de Faculdade São Salvador

** Mestre em Ciências da Saúde Universidade Estadual do Sudoeste da Bahia e docente da Faculdade Independente do Nordeste

*** Doutora em Enfermagem pela Universidade Federal da Bahia e docente da Universidade Federal da Bahia

**** Fisioterapeuta, doutora em Ciências da Saúde pela Universidade Federal do Rio Grande do Norte com estágio pósdoutoral em Saúde Coletiva pelo Instituto de Saúde Coletiva da Universidade Federal da Bahia. Docente da Universidade Estadual do Sudoeste da Bahia e docente da Faculdade Independente do Nordeste

\section{Resumo}

Objetivo: Este estudo tem por objetivo identificar os determinantes da sobrecarga e desconforto emocional em cuidadores de idosos com comprometimento da capacidade funcional. Materiais e Métodos: Trata-se de um estudo seccional realizado no município de Jequié/BA, com amostra de 40 cuidadores. O instrumento foi constituído de informações sociodemográficos, condições de saúde, Escala de Sobrecarga de Zarit e Self Reporting Questionnaire. Os dados foram analisados por meio de análise descritiva, testes do Mann Whitney e Kruskal-wallis, com $p<0,05$. Resultados: Verificou-se diferença estatística significativa entre o fator 1 com não ter recebido informações sobre a doença $(p=0,029)$ e o nível de escolaridade referente a analfabeto $(p=0,005)$; entre fator 2 com não ter recebido informações sobre a doença $(p=0,038)$, com ser o cuidador principal $(p=0,014)$ e estado civil separado, desquitado ou divorciado $(p=0,011)$; e entre o fator 4 com não ter recebido informações sobre a doença $(p=0,037)$ e estado civil separado, desquitado ou divorciado. Conclusão: Foi possível constatar que os cuidadores apresentavam com comprometimentos relacionados à saúde, predominando o desconforto emocional, que na maioria dos cuidadores apresentou pontuação acima da média.

Palavras-chave: Cuidado; Sobrecarga; Desconforto. 


\title{
DETERMINANTS OF OVERLOAD AND EMOTIONAL DISCOMFORT IN CAREGIVERS OF ELDERLY WITH FUNCTIONAL ABILITY IMPAIRMENT
}

\begin{abstract}
Objective: identify the determinants for overload and emotional discomfort in caregivers of elderly with functional ability impairment. Materials and methods: it's a sectional study made in Family Health Units in the municipality of Jequié/BA. The sample consisted of 40 caregivers of elderly with functional ability impairment. The instrument consisted of social-demographic information, health conditions, Zarit Overload Scale and the Self Reporting Questionnaire. The data was analized by decriptive analysis, tests of the Chi-square and Mann Whitney with $p<0,05$. Results: a significant statistic difference was established between factor 1 with not having received information about the illness $(p=0,029)$ and the scholar level referring to iliteracy $(p=0,005)$; between factor 2 with not having received information about the illness $(p=0,038)$, with being the main caregiver $(p=0,014)$ and civil status as separated, under separation or divorced $(p=0,011)$; and between factor 4 with not having received information about the illness $(p=0,037)$ and civil status as separated, under separation and divorced. Conclusions: the overload and emotional discomfort in caregivers of elderly with impairment of functional abilities suffer influence from variables like not having received information about the illness, scholar level referring to iliteracy, civil status as separated, under separation or divorced and being the main caregiver.
\end{abstract}

Keywords: Caution; Overload; Discomfort.

\section{INTRODUÇÃO}

A população de idosos vem aumentando em ritmo acelerado, apresentando demandas e necessidades emergenciais no campo da saúde e da sua assistência, principalmente quando esse grupo desenvolve incapacidades, necessitando de cuidadores para realização de suas atividades. ${ }^{(1)}$

O cuidador de idosos é o indivíduo responsável por oferecer ajuda e assistência naquilo que o idoso é incapaz de fazer sozinho, buscando melhorar direta ou indiretamente a qualidade de vida da pessoa cuidada. ${ }^{(2)}$

Esses cuidadores são submetidos, na maioria das vezes, a uma mudança na sua rotina habitual, passando a integrar e a ser parte fundamental da vida do idoso cuidado. Devido ao nível de envolvimento nos cuidados com o paciente, o cuidador muitas vezes é induzido a não prestar atenção na sua própria saúde, ocasionando uma sobrecarga e um desconforto emocional por negligência do autocuidar. ${ }^{(3)}$

Alteração do sono, nível elevado de estresse, diminuição dos vínculos sociais, insatisfação com a vida atual, presença de doenças crônicas e distúrbios músculos-esqueléticos são alguns dos problemas enfrentados por cuidadores, cuja frequência está associada diretamente ao aumento do grau de dependência do idoso. ${ }^{(4)}$

Diante desse quadro faz-se necessário o entendimento dos fatores que influenciam na relação entre cuidados próprios e cuidados ao idoso dependente, para que, a partir desses determinantes possam ser traçadas estratégias para melhora bidirecional entre esses dois grupos. Sendo assim, o objetivo desse estudo foi identificar os determi- 
nantes da sobrecarga e desconforto emocional em cuidadores de idosos com comprometimento da capacidade funcional.

\section{MATERIAIS E MÉTODOS}

Trata-se de um estudo seccional que analisou dados de uma pesquisa de intervenção de base domiciliar, denominada Relação entre capacitação dos cuidadores familiares e a qualidade da assistência prestada ao idoso com comprometimento da capacidade funcional. O local de estudo foi às residências dos idosos cadastrados nas Unidades de Saúde da Família da área de abrangência do bairro do Jequiezinho, no município de Jequié/BA.

A amostra do presente estudo foi constituída por 40 cuidadores de idosos com comprometimento da capacidade funcional, sendo esta pesquisa aprovada (Parecer $n^{\circ} .189 / 2008$ ) pelo Comitê de Ética em Pesquisa com Seres Humanos, da Universidade Estadual do Sudoeste da Bahia.

$O$ instrumento de pesquisa foi constituído de informações sociodemográficos, e condições de saúde. A sobrecarga no cuidador foi avaliada pela Escala de Sobrecarga de Zarit(5) e o desconforto emocional no cuidado foi avaliado pelo Self Reporting Questionnaire. ${ }^{(6)}$

A Escala de Zarit(5) tem 22 questões, cada questão é avaliada segundo uma escala tipo Likert com cinco possíveis respostas. O Autor que a validou e adaptou à população portuguesa, revela que este instrumento apresenta boa consistência interna (alfa de Cronbach de 0,93), salientando que a análise fatorial permite identificar 4 fatores criados empiricamente. Os fatores são: impacto da prestação de cuidados, relação interpessoal, expectativas face o cuidar e percepção de autoeficácia.

O Self Reporting Questionnaire( ${ }^{(6)}$ é um instrumento composto por 20 questões que avaliam os sintomas emocionais e físicos relacionados a quadros psiquiátricos como: ansiedade, depressão, reações psicossomáticas, irritação, cefaleia constante, queixas de insônia e cansaço mental, com escala de respostas dicotômicas. Quanto maior o número de respostas "sim" maior o nível de estresse emocional.

Os dados foram analisados no Programa Estatístico SPSS versão 20.0, sendo realizada análise descritiva e aplicação dos testes estatísticos do Qui-Quadrado de Pearson e Mann Whitney, com um nível de significância de 5\%.

\section{RESULTADOS}

Os cuidadores de idosos avaliados apresentaram média de idade de 55,35 $( \pm 11,98)$ anos. Sendo verificada uma maior frequência do sexo feminino $(85,0 \%)$, casados $(42,5 \%)$, com nível de escolaridade entre 5 a 8 anos de estudo (30,0\%), com grau de parentesco referente a ser o (a) filho (a) do idoso $(47,5 \%)$ e com problema de saúde $(82,5 \%)$, sendo mais frequente a hipertensão arterial associada à osteoporose $(17,5 \%)$.

Tabela 1 - Distribuição dos cuidadores segundo sexo, estado civil, escolaridade, grau de parentesco com o idoso e presença de problemas de saúde. Jequié/BA, 2016

(continua)

\begin{tabular}{lccc}
\hline & VARIÁVEIS & N & \% \\
\hline Sexo & 34 & 85,0 \\
\hline Feminino & 6 & 15,0 \\
Masculino & & \\
Estado Civil & 13 & 32,5 \\
Solteiro & 17 & 42,5 \\
Mora com esposo (a) ou companheiro (a) & 10 & 25,0 \\
Separado (a), desquitado (a) ou divorciado (a) & &
\end{tabular}


Tabela 1 - Distribuição dos cuidadores segundo sexo, estado civil, escolaridade, grau de parentesco com o idoso e presença de problemas de saúde. Jequié/BA, 2016

(conclusão)

\begin{tabular}{|c|c|c|}
\hline VARIÁVEIS & $\mathrm{N}$ & $\%$ \\
\hline \multicolumn{3}{|l|}{ Nível de Escolaridade } \\
\hline Analfabeto & 11 & 27,5 \\
\hline Sabe ler/escrever informal & 6 & 15,0 \\
\hline 1 a 4 anos & 5 & 12,5 \\
\hline 5 a 8 anos & 12 & 30,0 \\
\hline 9 a 12 anos & 4 & 10,0 \\
\hline 13 ou mais anos & 2 & 5,0 \\
\hline \multicolumn{3}{|l|}{ Grau de Parentesco } \\
\hline Filho & 19 & 47,5 \\
\hline Cuidador & 4 & 10,0 \\
\hline Irmã & 4 & 10,0 \\
\hline Conjugue & 7 & 17,5 \\
\hline Sobrinha & 3 & 7,5 \\
\hline Neta & 2 & 5,0 \\
\hline Nora & 1 & 2,5 \\
\hline \multicolumn{3}{|l|}{ Presença de Problemas de Saúde } \\
\hline Não & 7 & 17,5 \\
\hline Sim & 33 & 82,5 \\
\hline Total & 40 & 100,0 \\
\hline
\end{tabular}

Fonte: dados da Pesquisa.

$\mathrm{Na}$ avaliação do processo de cuidar, verificou-se que $90,00 \%$ são cuidadores principais, sendo que $67,50 \%$ não foram informados sobre a doença do idoso e 90,00\% não têm curso de cuidador. Observou-se que $88,90 \%$ trabalham no cuidado ao idoso 7 dias/semana, $44,44 \%$ com o tempo de 24 horas/ dia. Em relação ao recebimento de ajuda no pro- cesso do cuidado, $88,90 \%$ relatou não recebe ajuda de entidades sociais.

Em relação à sobrecarga do cuidador constatou-se que a maioria dos cuidadores apresentou pontuação acima do valor da média da pontuação do fator apenas no fator $1(55,00 \%)$ e no fator 2 $(62,50 \%)$.

Tabela 2 - Distribuição dos cuidadores segundo cuidador principal, informações sobre a doença e ter curso de cuidador. Jequié/BA, 2016

\begin{tabular}{lcc}
\hline \multicolumn{1}{c}{ VARIÁVEIS } & N & $\%$ \\
\hline Cuidador Principal & 36 & 90,00 \\
Sim & 4 & 10,00 \\
Não & & 32,50 \\
Informado sobre a doença do idoso & 13 & 67,50 \\
Sim & 27 & 10,00 \\
Não & & 90,00 \\
Tem curso de cuidador & 4 & 100,00 \\
$\quad$ Nim & 36 & 40 \\
\hline Total & & \\
\hline
\end{tabular}

Fonte: dados da Pesquisa. 
$\mathrm{Na}$ avaliação do desconforto emocional, os cuidadores obtiveram pontuação média de 6,60
$( \pm 4,60)$ pontos, sendo que a maioria dos cuidadores apresentou pontuação acima da média $(55,00 \%)$.

Tabela 3 - Distribuição dos cuidadores segundo a pontuação obtida nos fatores da escala de Zarit. Jequié/BA, 2016

N $\%$

\section{Fator 1 (Impactos da prestação de cuidados)}

-31,13 pontos

$<31,13$ pontos

Fator 2 (Relação interpessoal)

$\cdot 18,83$ pontos

$<18,83$ pontos

Fator 3 (Expectativas face o cuidar)

$\cdot 12,78$ pontos

$<12,78$ pontos

\section{Fator 4 (Percepção de auto-eficiência)}

\begin{tabular}{llc}
$\cdot 3,50$ pontos & 18 & 45,00 \\
$<3,50$ pontos & 22 & 55,00 \\
\hline Total & 40 & 100,00 \\
\hline
\end{tabular}

$22 \quad 55,00$

$25 \quad 62,50$

15

$19 \quad 47,50$

21

Fonte: dados da Pesquisa.

Com a aplicação do teste de Mann-Whitney entre as variáveis do estudo (sexo, faixa etária, presença de problemas de saúde, ser o cuidador principal, ter curso e ter sido informado sobre a doença) com as médias dos fatores da escala de Zarit e a média do Self Reporting Questionnaire, verificou-se que houve diferença estatística significativa entre o fator 1 e não ter recebido informações sobre a doença $(p=0,029)$; entre fator $2 \mathrm{com}$ não ter recebido informações sobre a doença $(p=0,038)$ e com ser o cuidador principal $(p=0,014)$; e entre o fator 4 e não ter recebido informações sobre a doença $(p=0,037)$.

Por meio da aplicação do teste de Kruskal-wallis entre as variáveis do estudo (estado civil, nível de escolaridade e grau de parentesco) com as médias dos fatores da escala de Zarit e a média do Self Reporting Questionnaire, constatou-se que houve diferença estatística significativa entre o fator $1 \mathrm{e}$ o nível de escolaridade referente a analfabeto $(p=$ $0,005)$; entre o fator 2 e estado civil separado(a), desquitado(a) ou divorciado(a) $(p=0,011)$; entre o fator 4 com estado civil separado(a), desquitado(a) ou divorciado(a) $(p=0,025)$ e a média do Self Reporting Questionnaire acima de 6,60 pontos $(p=0,036)$.

\section{DISCUSSÃO}

A investigação da relação cuidador e cuidado ao idoso é de fundamental importância para a área da geriatria/gerontologia e para a sociedade, principalmente nos países em processo de envelhecimento como é o caso do Brasil, visto que o aumento no número de idosos tem sido progressivo. ${ }^{(6,7)}$

No presente estudo observou-se que a média de idade dos cuidadores aproximava-se do limite de transição de adulto para idoso, este achado faz surgir uma nova realidade: idosos cuidando de idosos. Desta forma, pessoas que vivenciam o enveIhecimento, ou que passaram a vivenciar, acabam tendo que adquirir uma nova função, principalmente quando, o grau de parentesco é esposa ou filha mais velha. ${ }^{(8)}$ 
Outro fato preocupante em relação à caracterização dos cuidadores é que a maioria era do sexo feminino, casados, com nível de escolaridade entre 5 a 8 anos de estudo, com grau de parentesco referente a ser o(a) filho(a) do idoso e com problema de saúde, sendo mais frequente a hipertensão arterial associada à osteoporose. Estas características tornam esses cuidadores mais susceptíveis à ocorrência de desgaste físico e emocional, e a prestação de um cuidado de menor qualidade ao idoso. Esses dados de características dos cuidadores avaliados são semelhantes às características encontradas em estudo no qual verificou-se que houve uma maior distribuição de cuidadores do sexo feminino, com faixa etária de 22 a 77 anos, parentesco em relação ao idoso correspondente a filha, esposa e neta, tempo de estudo de um a onze anos, e com problemas de saúde. ${ }^{(9)}$

Um achado que chamou bastante atenção no nosso estudo foi perceber que embora a maior parte da população fosse composta por cuidadores principais, apenas uma minoria dos cuidadores tinha informações a respeito da doença do idoso e capacitação para prestação do cuidado. Estudos demonstram que o nível de instrução e preparação do cuidador melhora significativamente a vida dos idosos, e acrescenta que todo cuidador deve receber em suas residências, constantemente visitas de profissionais de saúde capacitados a fim de orientar como proceder desde as situações mais simples até as mais complicadas..$^{(10-12)}$

Vilela et al ${ }^{(11)}$ concluiu em seu estudo que grande parte dos cuidadores de idosos são únicos e que além de desempenharem essa função, têm sob seus cuidados, na família, dependentes como: filhos(as), netos(as) e outros parentes portadores de cuidados especiais. Esse dado também se fez presente em nosso estudo. A atividade de prestação de cuidado ao idoso com comprometimento da capacidade funcional é cansativo e exaustivo, podendo provocar o prejuízo da saúde mental e física do cuidador. Nesses casos, os cuidadores precisam de suporte social e familiar, de alguém que divida com ele as suas atividades, para que esse cuidador possa manter a própria saúde e poder cuidar de si mesmo. Não dispondo de tal suporte, os cuidadores ficam expostos a riscos de adoecer, não pelo cuidado em si, mas pela sobrecarga a que são submetidos. ${ }^{(11)}$

Atualmente são comuns as situações onde os membros da família não se encontram disponíveis, estão despreparados ou ficam sobrecarregados com a responsabilidade de cuidar dos idosos com comprometimento da capacidade funcional, e desta forma prestam suporte inadequado e ineficaz ao idoso. ${ }^{(13)}$ No presente estudo, a maior parte dos cuidadores não foi informada sobre a doença do idoso e nem tem formação para o cuidado ao idoso. Alguns estudos mostram que o nível de instrução interfere de forma significativa no processo de cuidar de idosos, especialmente nos casos de portadores de comprometimento da capacidade funcional, os quais necessitam de cuidados especiais e expõem o cuidador a estresse prolongado. $(7,8,14)$

Quanto ao tempo destinado ao cuidado observou-se que a maioria dos cuidadores trabalha no cuidado ao idoso 7 (dias) por semana, com o tempo de 24 (vinte e quatro) horas por dia. Essa jornada intensa de trabalho faz com que o cuidador deixe de realizar o seu autocuidado, se isole socialmente, apresente comprometimento de relações pessoais e fique suscetível ao acometimento por distúrbios psiquiátricos, como por exemplo, um quadro depressivo. Um estudo sobre o ato de cuidar constatou que os cuidadores dispensam a maior parte do dia ao cuidado prestado, não tendo tempo para desenvolver atividades de lazer e descanso, provocando assim no cuidador sobrecarga física e emocional. ${ }^{(15)}$

Segundo Schossler e Crossetti, ${ }^{(16)}$ as privações e a rotina do cuidador principal não permitem que as suas necessidades humanas sejam satisfeitas, fato que acontece devido à falta de outra pessoa para auxiliá-lo nas ações de cuidado ao idoso.

Em relação ao recebimento de ajuda no processo do cuidado verificou-se uma elevada frequência de cuidadores que relatou não receber ajuda de entidades sociais, dados estes corroborados por 
estudo realizado por Karsch, ${ }^{(10)}$ no qual observou-se que mais de $90 \%$ das famílias de idosos com comprometimento da capacidade funcional não recebem ajuda de serviços, grupos voluntários ou agências particulares.

O apoio social é de extrema importância para estes cuidadores de idosos com comprometimento da capacidade funcional, pois representam a oportunidade de atendimento das necessidades sociais básicas obtidas através da interação com os outros. Essas necessidades incluem afeto, estima, pertença, identidade e segurança e são satisfeitas através da ajuda emocional e instrumental, sendo que a primeira engloba a compreensão, o afeto e a estima, e a segunda o aconselhamento, a informação e a assistência material. ${ }^{(11)}$

Em relação à sobrecarga do cuidador constatou-se que a maioria dos cuidadores apresentou pontuação acima do valor da média da pontuação nos fatores da escala de Zarit, (5) apenas nos fatores referentes aos impactos da prestação de cuidados e relação interpessoal. Vários autores confirmam que o cuidado informal, além de ser realizado, principalmente, por pessoas com vínculo de parentesco, também é centrado em um único cuidador familiar, o qual se sobrecarrega, em muitos casos, com tal responsabilidade. ${ }^{(17-19)}$

A atividade de cuidar de pessoas idosas, muitas vezes, gera sobrecarga na vida do cuidador, essa sobrecarga pode acarretar o desenvolvimento de sintomas psiquiátricos, fadiga, uso de medicamentos psicotrópicos, além de ter sua própria saúde prejudicada, o que leva à falta de condições para cuidar do idoso.

$\mathrm{Na}$ avaliação do desconforto emocional, a maior parte dos cuidadores obteve pontuação média elevada, demonstrando assim que estes cuidadores apresentam comprometimento emocional decorrente da atividade de cuidado ao idoso. A condição de cuidador pode causar situações de estresse, desgaste físico-emocional, perda da identidade e isolamento social. Cuidar de idosos dependentes pode acarretar angústia, solidão, tensão, tristeza, alterações no bem-estar do cuidador a nível da sua saúde, da vida social, da disponibilidade econômica, da rotina familiar, do desempenho profissional, levando ao aparecimento de níveis elevados de sobrecarga.

Assim, é importante que opções assistenciais sejam oferecidas aos cuidadores com o objetivo de adequar a assistência às necessidades de saúde/ doença tanto do idoso quanto do cuidador, melhorando assim a qualidade de vida de ambos. Logo é fundamental que os profissionais de saúde, em especial os de saúde mental, estejam efetivamente atentos à análise do contexto familiar no qual este cuidador encontra-se inserido, a fim de minimizar o desgaste emocional e melhorar o estado de saúde dos cuidadores.

No presente estudo, como determinantes de sobrecarga e desconforto emocional nos cuidadores o nível de escolaridade analfabeto, com o fato de não ter recebido informações sobre a doença apresentada pelos idosos e por apresentar estado civil separada. O nível educacional mais baixo dos cuidadores está associado ao maior número de queixas de esforço físico e desgaste emocional, dados estes corroborados por estudo no qual os idosos com menores anos de escolaridade foram diagnosticados com sobrecarga intensa. ${ }^{(20,21)}$

Esta situação pode gerar no cuidador sentimentos de despreparo e impotência, que têm uma carga de ordem emocional tão acentuada que pode repercutir diretamente no desempenho do papel e na qualidade do cuidado prestado.

Outros determinantes de sobrecarga e desconforto emocional nos cuidadores encontrados no presente estudo foi que a relação pessoal apresentou diferença estatística significativa com o fato de ser o cuidador principal e de estado civil separado. A necessidade de prestar os cuidados de forma sistemática e a tensão provocada pela complexidade que os envolve podem tornar os cuidadores pacientes em potencial, pois muitas vezes esquecem de cuidar de si para cuidarem dos idosos e de suas relações pessoais, relegando-se a um segundo plano para cumprirem uma rotina estafante de cuidados. Dados estes confirmados em estudo 
realizado em Fortaleza onde encontrou-se a associação da sobrecarga implicações na vida pessoal do cuidador com o fato de ser cuidador principal e ser solteiro. ${ }^{(22)}$

\section{CONCLUSÃO}

Constatou-se no presente estudo uma maior distribuição de cuidadores de idosos do sexo feminino, casados, com nível de escolaridade entre 5 a 8 anos de estudo, com grau de parentesco referente a ser o(a) filho(a) do idoso e com problema de saúde, sendo mais frequente a hipertensão arterial associada à osteoporose. Verificou-se ainda que a maioria são cuidadores principais, sendo que não foram informados sobre a doença do idoso e não têm curso de cuidador. Estes cuidadores e sua maior parte trabalham no cuidado ao idoso 7 (dias) por semana, com o tempo de 24 (vinte e quatro) horas por dia.

Em relação a sobrecarga do cuidador observou-se que a maioria dos cuidadores apresentou pontuação elevado nos fatores 1 e $2 . \mathrm{Na}$ avaliação do desconforto emocional, a maior parte dos cuidadores apresentou pontuação acima da média. Observou-se diferença estatística significativa entre o fator 1 com não ter recebido informações sobre a doença e o nível de escolaridade referente a analfabeto; entre fator 2 com não ter recebido informações sobre a doença, com ser o cuidador principal e estado civil separado, desquitado ou divorciado; e entre o fator 4 com não ter recebido informações sobre a doença e estado civil separado, desquitado ou divorciado.

\section{REFERÊNCIAS}

1. Seima MD, Lenardta MH. Sobrecarga do cuidador familiar de idoso com Alzheimer. Texto \& Contexto Enf. 2011 [acesso em 2015 set 3O]; 1O(2):388 - 398. Disponível em: file:///C:/Users/ LucianaReis/Downloads/9901-37818-1-PB.pdf
2. Rocha MPF, Vieira MA, Sena RR. Desvelando o cotidiano dos cuidadores informais de idosos. Rev Bras Enferm. 2008 [acesso em 2015 set 30]; 61(6): 801-8. Disponível em: http://www. scielo.br/pdf/reben/v6ln6/aO2v6ln6

3. Rocha Júnior PR et. al. Efeito da capacitação dos cuidadores informais sobre a qualidade de vida de idosos com déficit de autocuidado. Ciênc Saúde Coletiva. 2011 [acesso em 2015 set 30]; 16(7): 3131-38. Disponível em: http://www. scielo.br/pdf/csc/v16n7/13.pdf

4. Silveira TM, Caldas CP, Carneiro TF. Cuidando de idosos altamente dependentes na comunidade: um estudo sobre cuidadores familiares principais. Cadernos de Saúde Pública 2006 [acesso em 2015 set 30 ]; 22(8):1629-38. Disponível em: http:// www.scielo.br/scielo.php?pid=SO1O2$311 \times 2006000800011 \&$ script=sci_ abstract\&tlng=pt

5. Zarit SH. Research perspective on family caregiving. In: Cantor M. Family caregiving: Agenda for the future. San Francisco: American Society for Aging; 1994. p. 9-24.

6. Gonçalves DM, Stein AT, Kapczinski F. Avaliação de desempenho do SelfReporting Questionnaire como instrumento de rastreamento psiquiátrico: um estudo comparativo com o Structured Clinical Interview for DSM-IV-TR. Cad Saúde Pública. 2008 [acesso em 2015 set 30];24(2): 380390. Disponível em: http://www.scielo.br/ scielo.php?script=sci_arttext\&pid=SO1O2$311 \times 2008000200017$

7. Moreira MD, Caldas CP. A importância do cuidador no contexto da saúde do idoso. Esc Anna Nery Rev Enferm. 2007[acesso em 2015 set 30];11(3):520-5. Disponível em: http://www. redalyc.org/pdf/1277/127715309019.pdf

8. Amendola F, Oliveira MAC, Alvarenga MRM. Influence of social support on the quality of life of family caregivers while caring for people with dependence. Rev Esc Enferm USP. 2011 [acesso em 2015 set 30];45(4): 884-9. Disponível em: http://www.producao.usp.br/ bitstream/handle/BDPI/3758/art_AMENDOLA_ Influencia_do_apoio_social_na_qualidade_de_2O11. pdf? sequence $=1$ 
9. Guedea MTD et. al. Necessidade de apoio social em cuidadores de familiares idosos mexicanos. Psicol Soc. 2009 [acesso em 2015 set 30]; 21(2): 242-49. Disponível em: http:// www.scielo.br/pdf/psoc/v2ln2/v2ln2all.pdf

10. Karsch UM. Idosos dependentes: famílias e cuidadores. Cad Saúde Pública. 2003 [acesso em 2015 set 30]; 19(3):861-6. Disponível em: http://www.scielo.br/scielo.php?script=sci_ arttext\&pid=SO1O2-311X2003000300019

11. Vilela ABA et al. Perfil do familiar cuidador de idoso doente e/ou fragilizado no contexto sociocultural de Jequié-BA. Rev Bras Geriatr Gerontol. 2006 [acesso em 2015 set 30];9(1):55-69. Disponível em: http://pesquisa. bvs.br/brasil/resource/pt/lil-450307

12. Souza LM, Wegner W, Gorini MLPC. Educação em saúde: uma estratégia de cuidados ao cuidador leigo. Rev Lat Am Enferm. 2007 [acesso em 2015 set 30];15 (2): 337-43. Disponível em: http://www.scielo.br/pdf/rlae/ v15n2/pt_v15n2a22.pdf

13. Nardi EFR, Oliveira MLF. Significado de cuidar de idosos dependentes na perspectiva do cuidador familiar. Ciênc Cuid Saúde. 2009 [acesso em 2015 set 30]; 8(3):428-35. Disponível em: http://www.scielo.br/pdf/tce/ v25nl/0104-0707-tce-25-01-0760014.pdf

14. Veras R. Population aging today: demands, challenges and innovations. Rev Saude Publica 2009 [acesso em 2015 set 30]; 43:548-54. Disponível em: http:// www.scielo.br/scielo.php?pid=SOO34$89102009000300020 \&$ script=sci_ arttext\&tlng=en

15. Rodríguez-Sánchez E et. al. Relationships between quality of life and family function in caregiver. BMC Fam Pract. 2011 [acesso em 2015 set 30];12:1-7. Disponível em: http://bmcfampract.biomedcentral.com/ articles/10.1186/1471-2296-12-19
16. Schossler T, Crossetti MGO. Cuidador Domiciliar do Idoso e o Cuidado de Si: uma análise através da Teoria do Cuidado Humano de Jean Watson. Texto \& Contexto Enferm. 2008;17(2):280-287. Disponível em: http://www. scielo.br/pdf/tce/v17n2/O9.pdf

17. Reis LA et al. Avaliação do suporte familiar em idosos residentes em domicílio. Aval Psicol. 2011 [acesso em $2 \mathrm{Ol5}$ set 30]; 10 (2). Disponível em: http://pepsic.bvsalud.org/scielo.php?script=sci_ar ttext\&pid=S1677-04712011000200002

18. Zem-Mascarenhas SH, Barros ACT, Carvalho SJC. Um olhar atento sobre a prática do cuidador familiar. REME: Rev Min Enferm. 2006 [acesso em 2015 set 30];10(2):132-37. Disponível em: http://reme.org.br/artigo/detalhes/397

19. Mendes GD, Miranda SM, Borges MMMC. Saúde do cuidador de idosos: um desafio para o cuidado. Revista de Enfermagem Integrada 2010 [acesso em 2015 set 30]; 3(1):408-

21. Disponível em: http://www.unilestemg.br/ enfermagemintegrada/artigo/v3/O4-saudecuidador-idosos-desafio.pdf

2O. Rodrigues SLA, Watanabe HAW, Derntl AM. A saúde de idosos que cuidam de idosos. Rev Esc Enferm USP. 2006 [acesso em 2015 set 30];4O(4):493- 500. Disponível em: http://www. scielo.br/pdf/reeusp/v4On4/v4On4aO6.pdf

21. Ferreira CG, Alexandre TS, Lemos ND. Fatores associados à qualidade de vida de cuidadores de idosos em assistência domiciliar. Saúde Soc. 2011 [acesso em 2015 set 30];2O(2):398-409. Disponível em: http://www.scielo.br/pdf/sausoc/ v2On2/12.pdf

22. Martins JJ. Necessidades de educação em saúde dos cuidadores de pessoas idosas. Texto \& Contexto Enf. [acesso em 2015 set 30] 2007;16(2):254-62. Disponível em: http://www. scielo.br/pdf/tce/v16n2/aO7v16n2 